\title{
An evaluation of the characteristics and outcomes of users of a street triage service: retrospective case note review
}

\author{
Andrew Molodynski, ${ }^{1,2}$ Sarah Hollingsworth, ${ }^{1}$ Louise Penzenstadler, ${ }^{1,3}$ Devon Perfect, ${ }^{1}$ \\ Stephen Puntis ${ }^{2}$ (1)
}

BJPsych Bulletin (2019) 43, 290-294, doi:10.1192/bjb.2019.32

\begin{abstract}
1Oxford Health NHS Foundation Trust; ${ }^{2}$ Oxford University; ${ }^{3}$ Geneva University Hospitals

Correspondence to Andrew Molodynski (andrew.molodynski@oxfordhealth.nhs. uk)

First received 15 Oct 2018, final revision 7 Mar 2019, accepted 27 Mar 2019

(c) The Authors 2019. This is an Open Access article, distributed under the terms of the Creative Commons Attribution-NonCommercialNoDerivatives licence (http:// creativecommons.org/licenses/by-ncnd/4.0/), which permits noncommercial re-use, distribution, and reproduction in any medium, provided the original work is unaltered and is properly cited. The written permission of Cambridge University Press must be obtained for commercial re-use or in order to create a derivative work.
\end{abstract}

\begin{abstract}
Aims and method Street triage services are now common but the population they serve is poorly understood. We aimed to evaluate a local service to determine the characteristics of those using it and their outcomes in the 90 day period following contact.

Results We found that there were high levels of service use and that the vast majority of contacts were via telephone rather than in person. Street triage was used by both existing secondary mental health patients and non-patients. Follow-up rates with secondary services were high in the former and low in the latter case.

Implications Services are very busy where they exist and may be replacing traditional crisis services. It is not apparent that they work to increase follow-up among those using them, unless they are already in contact with services. In this service, although there was a joint response model nearly all responses were provided by telephone.
\end{abstract}

Declaration of interest None.

Keywords Street triage; crisis care; community mental health teams.

\section{Background}

So-called 'street triage' services were first piloted in the UK several years ago in order to support police officers responding to mental health crises. Rates of use of section 136 of the Mental Health Act had been causing concern and the police service had reported an increasing number of contacts with a mental health component, with estimates ranging from 2 to $50 \%,{ }^{1,2}$ alongside a perceived lack of skills to manage such situations among officers. ${ }^{3}$ There were also growing concerns regarding the experience and rights of individuals, ${ }^{4}$ with several cases of vulnerable individuals spending long periods in police cells, including children. ${ }^{5}$

Although the introduction of street triage services was considered a logical and positive response to these issues, it was not based upon empirical evidence. It is extremely difficult to rigorously examine the effectiveness of such complicated interventions once they have been widely introduced, and traditional methods of gathering high-level evidence, such as randomised controlled trials, are very challenging indeed. This has been seen in previous studies of mental health law. ${ }^{6}$

Triage services vary significantly, both between and within areas. All offer a co-response model in which police and health staff work together to manage mental health crises. In some services this involves the mental health worker (usually a nurse or social worker) attending incidents with the police, in others there is real-time telephone advice, and in some both are available.

There remains scarce evidence for processes and outcomes in street triage services despite some high-quality local evaluations. ${ }^{7-9}$ A recent systematic review concluded that there is a lack of evidence for the effectiveness of street triage services and regarding the characteristics, experiences and outcomes of those who use them. It also concluded that there is substantial variation in the services offered. ${ }^{10}$ These gaps in knowledge need to be remedied to provide the most effective services for this vulnerable group. As the funding for many pilots comes towards an end, crucial questions remain about what these services should look like, who they should serve, what they achieve and who should fund them. This service evaluation aimed to provide data regarding the characteristics of street triage users and their outcomes in terms of service use over a 90 day period following initial contact.

\section{Method}

\section{Setting, design and participants}

We conducted a retrospective case note review of the co-response street triage service provided by Thames 
Valley Police and Oxford Health NHS Foundation Trust. This service covers the whole of Oxfordshire (680 000 population) and runs from $18.00 \mathrm{~h}$ to $04.00 \mathrm{~h}, 7$ days a week. The team comprises one police officer and one mental health worker per shift and offers both a ride-along mobile unit (where police officer and mental health worker are dispatched in a marked police car) and a control-room-based telephone triage service located at the local police station. At the time of the evaluation, the mobile unit was only available within Oxford city. Potential cases are referred to the street triage team either through the police control room or directly from police officers who are attending incidents. The service has been in operation since August 2014.

We collected data retrospectively for individuals who had contact with the street triage team between the months of April and June 2017 (3 months in total). Records were examined for the 90 days following initial contact. Data was collected from both National Health Service (NHS) (carenotes) and street triage service records. Each contact made with the street triage team is logged by a trained mental health worker in a Microsoft Excel spreadsheet as part of routine clinical practice. The information captured includes the referral source, service user demographics, notes describing the reason for the call, the response of the street triage team, and the outcome of the response. This information was coded by one member of our team (D.P.), and the codes were then checked by another (S.P.).

We also collected data on any previous contact with mental health services in the past year, previous detention under the mental health act at any time of life, and any history of drug or alcohol misuse or dependence. This study was part of a commissioned service evaluation for Thames Valley Police and Oxford Health NHS Foundation Trust and therefore no ethical approval was needed.

\section{Analysis}

We used descriptive statistics to summarise data on demographic and clinical characteristics and service use in our sample. We categorised individuals as either 'single' or 'multiple' street triage users based on their number of triage events over the 90 day follow-up and used cross tabulations to examine the relationships within the data. We used $t$-tests for continuous data and $\chi^{2}$-tests or Fisher's exact tests for categorical variables to compare demographic and clinical characteristics and service use between single and multiple users of street triage. We did not make adjustments for multiple testing and so our findings assume significance at the $P<0.05$ level.

\section{Results}

\section{Characteristics}

During the 3 month period analysed, there were 374 recorded incidents of street triage contact involving 307 different individuals. Of the 307 individuals, 244 (79\%) had a mental health record with Oxford Health NHS Foundation Trust. Demographic and clinical details of our sample are given in Table 1.

\begin{tabular}{|c|c|c|c|}
\hline & $N($ mean) & $\%($ s.d.) & Missing \\
\hline Age & 35.1 & 15.24 & $10(3.3 \%)$ \\
\hline Gender, male & 170 & 55 & $5(1.6 \%)$ \\
\hline Ethnicity & & & $138(45 \%)$ \\
\hline White & 147 & 87 & \\
\hline Black & 6 & 4 & \\
\hline Asian & 9 & 5 & \\
\hline Mixed & 5 & 3 & \\
\hline Other & 2 & 1 & \\
\hline In TVP area, yes & 237 & 98 & $64(21 \%)$ \\
\hline Primary diagnosis & & & $63(21 \%)$ \\
\hline None recorded & 42 & 17 & \\
\hline Non-affective psychoses & 39 & 16 & \\
\hline Affective psychoses & 13 & 5 & \\
\hline Affective disorders & 48 & 20 & \\
\hline $\begin{array}{l}\text { Post-traumatic stress } \\
\text { disorder }\end{array}$ & 12 & 5 & \\
\hline Eating disorders & 2 & 1 & \\
\hline Personality disorders & 47 & 19 & \\
\hline Developmental disorders & 22 & 9 & \\
\hline Other & 19 & 8 & \\
\hline IMD decile ${ }^{\mathrm{a}} 1$ most deprived & & & $64(21 \%)$ \\
\hline $1-3$ & 70 & 26 & \\
\hline $4-6$ & 83 & 3 & \\
\hline $7-10$ & 96 & 40 & \\
\hline Any previous MH use, yes & 222 & 72 & \\
\hline In past 2 weeks? & 118 & 38 & \\
\hline Within past year? & 191 & 62 & \\
\hline Previous MHA detention $^{\text {b }}$ & 67 & 22 & \\
\hline${\text { Previous ST } \text { contact }^{c}}$ & 82 & 27 & $65(21 \%)$ \\
\hline
\end{tabular}

a. Higher decile equals higher deprivation.

b. Previous mental health detention in Oxford Health only.

c. Previous ST contact on Oxford Health electronic health record only.

$\mathrm{IMD}$, index of multiple deprivation; $\mathrm{MH}$, mental health; MHA; Mental Health Act; TVP, Thames Valley Police.

The reasons recorded for the involvement of the street triage service are illustrated in Fig. 1.

\section{Outcome}

In 284 episodes (91\%), telephone advice was given either to the officer or the service user, with only $17(6 \%)$ calls resulting in face-to-face contact. Only nine contacts (3\%) resulted in section 136 use, with 199 (61\%) resulting in signposting to other services or no action, 30 (10\%) in accident and emergency department attendance, and $73(24 \%)$ in police intervention regarding an alleged/ actual offence. Longer-term outcomes are shown in Table 2.

Although overall $45 \%$ of people accessed mental health services in the 90 days following their initial contact, this 
Figure 1. Documented reasons for street triage involvement $\mathrm{ST}$, street triage; AWOL, absent without leave (from ward).

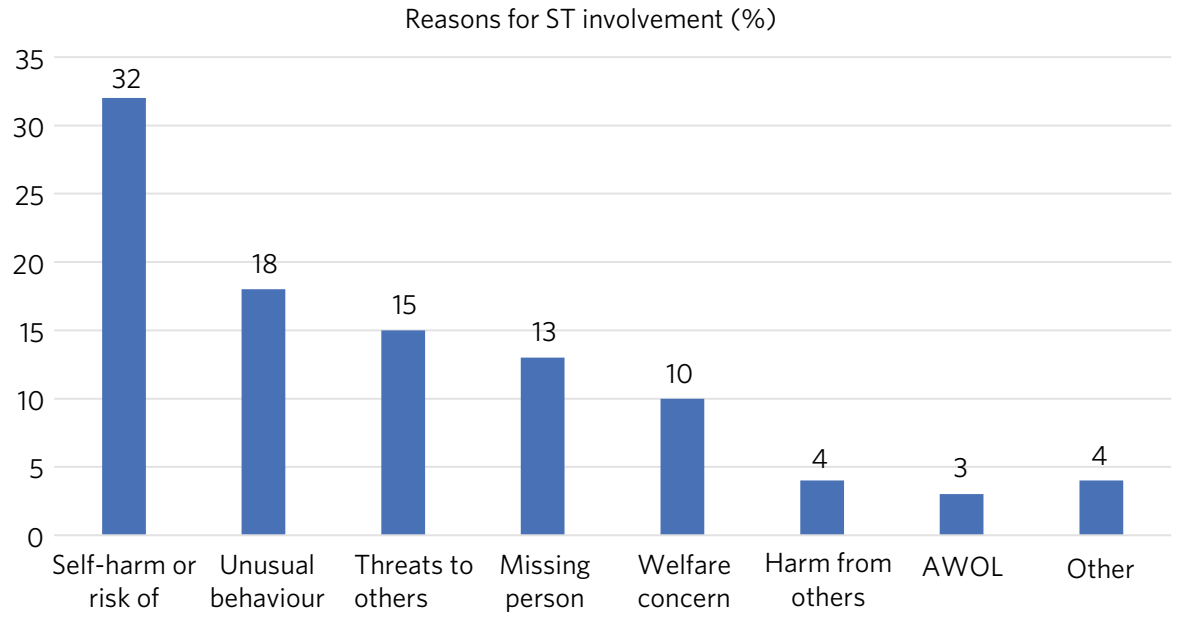

Reasons for ST involvement (\%)

rate varied substantially by whether and when they had had previous contact. These variations are discussed below.

\section{Single and repeat users}

Within the follow-up period, 257 people (84\%) only had one contact with street triage services, while 50 (16\%) had two or more. Of the 50 multiple attenders, 33 had one further contact and 17 had two or more. The maximum number of street triage contacts was 11 in the time period, with three individuals having more than five contacts. The peak time for repeat contacts was within the first 2 weeks. Single and multiple users were similar demographically and in terms of the response they received. There was a non-significant trend towards differences in diagnosis between the two groups $\left(\chi^{2}(4)=8.39, P=0.078\right)$, with non-affective psychoses ( $22 v$. $14 \%$ ) and personality disorders (31 v. 16\%) being more common in repeat attenders. Repeat users also had significantly higher levels of service use in the prior 2 weeks $(62 v .34 \%$; $\left.\chi^{2}(2)=12.85, P<0.001\right)$, in the past year $\left(86 v .58 \% ; \chi^{2}(2)=\right.$ $13.19, P<0.001)$ and in their lifetime (96 v. 68\%; $\chi^{2}(2)=$ 15.36, $P<0.001)$. They were more likely to access mental health services following the street triage contact (70 v. $\left.41 \% ; \chi^{2}(2)=13.39, P<0.001\right)$ and to be admitted to a psychiatric hospital $\left(22\right.$ v. 10\%; $\left.\chi^{2}(2)=5.77, P=0.016\right)$.

\section{Substance misuse}

Amongst those referred to street triage services, 88 (36\%) had a history of substance misuse (Table 3). In this group, $28(32 \%)$ of people had a diagnosis of dependence in their NHS records and the remainder had symptoms of misuse. The number of health problems related to alcohol use disorder was high. Over half of substance misuse cases involved alcohol (58\%), and 11 (13\%) of the overall substance misuse sample $(n=88)$ had mixed disorders involving alcohol and another substance. Only 38 (43\%) of individuals with a history of substance use disorder required street triage as a direct result of intoxication.

\section{Limitations}

This is a retrospective case note review within a short time frame and with a relatively short follow-up, so the results need to be examined with caution. However, our sample was large with few missing data on important indicators. Missing data were mostly due to the individual not having a mental health record with Oxford Health NHS Foundation Trust; therefore, the data were not missing at random as the two groups (those known to the mental health trust and those not known) were likely to be different in terms of demographics, clinical presentation, substance

\begin{tabular}{|lcc|}
\hline Table 2 Service users' outcomes within 90 days of index street triage (ST) incident, $n=307$ & $\%$ \\
\hline Admitted to hospital as in-patient, yes & $n$ & 13.0 \\
\hline Accessed mental health services, yes & $39^{\text {a }}$ & 45.3 \\
\hline If seen in the 2 weeks prior to ST incident, $n=118$ & 139 & 74.5 \\
\hline If not seen in the 2 weeks prior to ST incident, $n=189$ & 88 & 26.9 \\
\hline If seen in the year prior to ST incident, $n=191$ & 51 & 65.4 \\
\hline If not seen in the year prior to ST incident, $n=116$ & 125 & 12.1 \\
\hline All cause mortality & 14 & 2.6 \\
\hline Subject to a Section 136, yes & 8 & 7.2 \\
\hline
\end{tabular}

a. Five $(1.7 \%)$ participants were in-patients at time of street triage incident.

b. This metric only includes deaths recorded in Oxford Health's clinical records. 
Table 3. Substance misuse in sample, $n=307$

\begin{tabular}{lccc} 
& Missing (\%) & $n$ & $\%$ \\
\hline Recorded substance use disorder & $62(20)$ & 88 & 35.9 \\
\hline Misuse & & 60 & 68.1 \\
\hline Dependence & & 28 & 31.8 \\
\hline Type of substance misuse & $62(20)$ & & \\
\hline Alcohol & & 40 & 45.5 \\
\hline Alcohol and another substance & 11 & 12.5 \\
\hline $\begin{array}{l}\text { Intoxication apparent during index } \\
\text { ST incident }\end{array}$ & $0(0)$ & 38 & 12.4 \\
\hline
\end{tabular}

ST, street triage.

misuse and service use. We were not able to collect data from other services such as primary care or those for substance misuse.

\section{Discussion}

Street triage services are a major part of emergency care, with on average 100 contacts per month for a population of 680000 people. The majority of people (84\%) who use these services do so only once within a 90 day time period, with a minority using them repeatedly. Repeated use tended to be within 2 weeks, fitting with previous assumptions of a crisis-driven model of contact. Reasons for accessing triage services predominantly related to concerns for the individual's welfare due to self-harm, unusual behaviour or generalised concerns, with these making up three-quarters of contacts. The response in $94 \%$ of cases was by telephone, with a surprisingly small number of face-to-face contacts (this is a service resourced and set up for a mixed response as needed). As models vary significantly around the country and as a previous survey showed a clear preference among police officers for a face-to-face response, ${ }^{2}$ there is a need to understand the reasons for and outcomes of differing response styles.

Multiple users were more likely to have a diagnosis of psychosis or personality disorder and were significantly more likely to have used services before, with $96 \%$ having documented previous use. They were also significantly more likely to use services afterwards, including in-patient care. This information could be useful in longer-term planning for this group, with repeat street triage use being an indicator of higher need and an automatic trigger for a response from mental health services. Such intervention might mitigate the risks and reduce rates of hospital admission in this clearly vulnerable group. Such vulnerability is shown by the eight deaths in our sample population. It is important to note, however, that the population using the triage service is not composed only of mental healthcare patients, with one in four users having had no previous contact with secondary mental health services (at least within Oxford Health NHS Foundation Trust).

Thirty-six per cent of incidents involved documented substance misuse, perhaps less than might be expected given the association between crisis situations and substance misuse. Most police officers (65\%) are quite confident in determining intoxication most or all of the time, ${ }^{11}$ so underreporting seems unlikely to be a major issue. It remains important to recognise substance misuse, as many with these problems are not in specialised care and it is an added risk factor, whether the individual remains in the community or is detained in custody. ${ }^{12}$

While $45 \%$ of individuals accessed mental health services within 90 days, only $11 \%$ were admitted to hospital, and even fewer (7\%) were detained under section 136 . The very significant differences in subsequent access to mental healthcare between those who were already patients (75\%) and those who were not (9\%) are perhaps concerning as they suggest that street triage may not provide a fluid pathway into mainstream mental health services as hoped.

\section{Conclusions}

Street triage services are heavily used where available with a tendency to use a telephone model response by default in this area. Most referrals were made owing to concerns for individual welfare, and the majority of people used the service only once in 90 days.

The large differences in follow-up rates between current or recent patients and non-patients are concerning, as they suggest that triage services may not be adequately fulfilling one of their roles - as a pathway to mainstream services for this vulnerable group. Although one cannot draw causal conclusions from our data, they indicate a clear need for research studies that can more accurately determine access to services and patterns of care of those accessing triage services. This should in turn inform decision-making regarding these services. A crucial part of this is to directly ascertain the views and experience of the people who come into contact with such services at times of crisis alongside those of serving officers and the triage staff themselves. Do these services already, or will they, replace traditional crisis services, or will they wither as funding dries up? At present we simply do not know enough to take a view, let alone direct practice.

\section{About the authors}

Andrew Molodynski is a consultant psychiatrist at Oxford Health NHS Foundation Trust and Oxford University, and is BMA Consultant Committee Mental Health Lead, UK; Sarah Hollingsworth is a researcher at Oxford Health NHS Foundation Trust, UK; Louise Penzenstadler is a senior psychiatrist Geneva University Hospitals, Switzerland; Devon Perfect is a research assistant at Oxford Health NHS Foundation Trust; and Stephen Puntis is a postdoctoral researcher in the Department of Psychiatry at Oxford University, UK.

\section{References}

1 College of Policing. College of Policing Analysis: Estimating Demand on the Police Service. College of Policing, 2015.

2 Kirubarajan A, Puntis S, Perfect D, Tarbit M, Buckman M, Molodynski A. Street triage services in England: service models, national provision and the opinions of police. BJPsych Bulletin 2018; 42(6): 253-7.

3 Wells W, Schafer JA. Officer perceptions of police responses to persons with a mental illness. Policing 2006; 29(4): 578-601. 
4 Riley G, Freeman E, Laidlaw J, Pugh D. 'A frightening experience': detainees' and carers' experiences of being detained under Section 136 of the Mental Health Act. Med Sci Law 2011; 51(3): 164-9.

5 Justice Inspectorate 2015. The Welfare of Vulnerable People in Police Custody. HMIC, 2015 (https://www.justiceinspectorates.gov.uk/ hmicfrs/wp-content/uploads/the-welfare-of-vulnerable-people-in-policecustody.pdf).

6 Burns T, Rugkåsa J, Molodynski A, Dawson J, Yeeles K Vazquez-Montes $M$, et al. Community treatment orders for patients with psychosis (OCTET): a randomised controlled trial. Lancet 2013; 381(9878): 1627-33

7 Irvine AL, Allen L, Webber MP. Evaluation of the Scarborough, Whitby and Ryedale Street Triage Service. Research Report. Department for Social Policy and Social Work, University of York, 2016.

8 Keown $\mathrm{P}$, et al. Too much detention? Street Triage and detentions under Section 136 Mental Health Act in the North-East of England: a descriptive study of the effects of a Street Triage intervention. BM Open 2016; 6(11): e011837.

9 Jenkins O, Dye S, Obeng-Asare F, Nguyen N, Wright N. Police liaison and section 136: comparison of two different approaches. BJPsych Bulletin 2017; 41(2): 76-82.

10 Puntis S, Perfect D, Kirubarajan A, Bolton S, Davies F, Hayes A, et al. A systematic review of co-responder models of police mental health 'street' triage. BMC Psychiatry 2018; 18: 256.

11 Hollander Y, Lee SJ, Tahtalian S, Young D, Kulkarni J. Challenges relating to the interface between crisis mental health clinicians and police when engaging with people with a mental illness. Psychiatry Psychol Law 2012 19(3): 402-11

12 Norfolk GA. Deaths in police custody during 1994: a retrospective analysis. J Clin Forensic Med 1998; 5(1): 49-54.

$\left(\begin{array}{l}\text { OPEn } \\ \text { ACCESS }\end{array}\right.$ 